Behavioural Sciences | Professor Fumiko Satoh

\section{Double-suicide in modern Japan:}

An ageing society's crisis

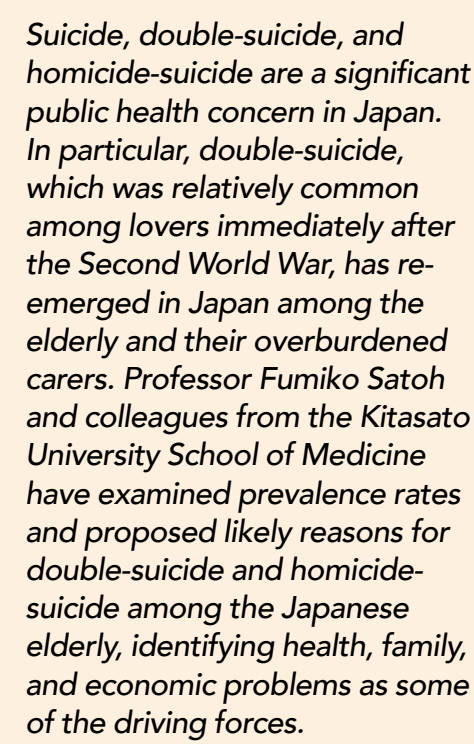

Ouble-suicide, particularly among couples, was a significant public
health concern in Japan after Second World War, when arranged marriages stopped being the norm and people began to marry partners of their choice. Nevertheless, in some cases, sway, leading couples to take their lives in fear they wouldn't be able to be togethe Although social norms have since relaxed contemporary Japan is seeing a return of this phenomenon. Japan, currently ranked seventh in suicide incidence rates worldwide, is grappling with an ageing society where the burden of care over the frail leads to caregiver exhaustion that may result in violence. With this in mind, the government is taking measures to understand the scale and address the mechanisms behind double-suicide and homicide-suicides, which, to-date, rema poorly understood. Fumiko Satoh and colleagues from the Kitasato University School of Medicine have examined explanters for douposed any the elderly in Japan.

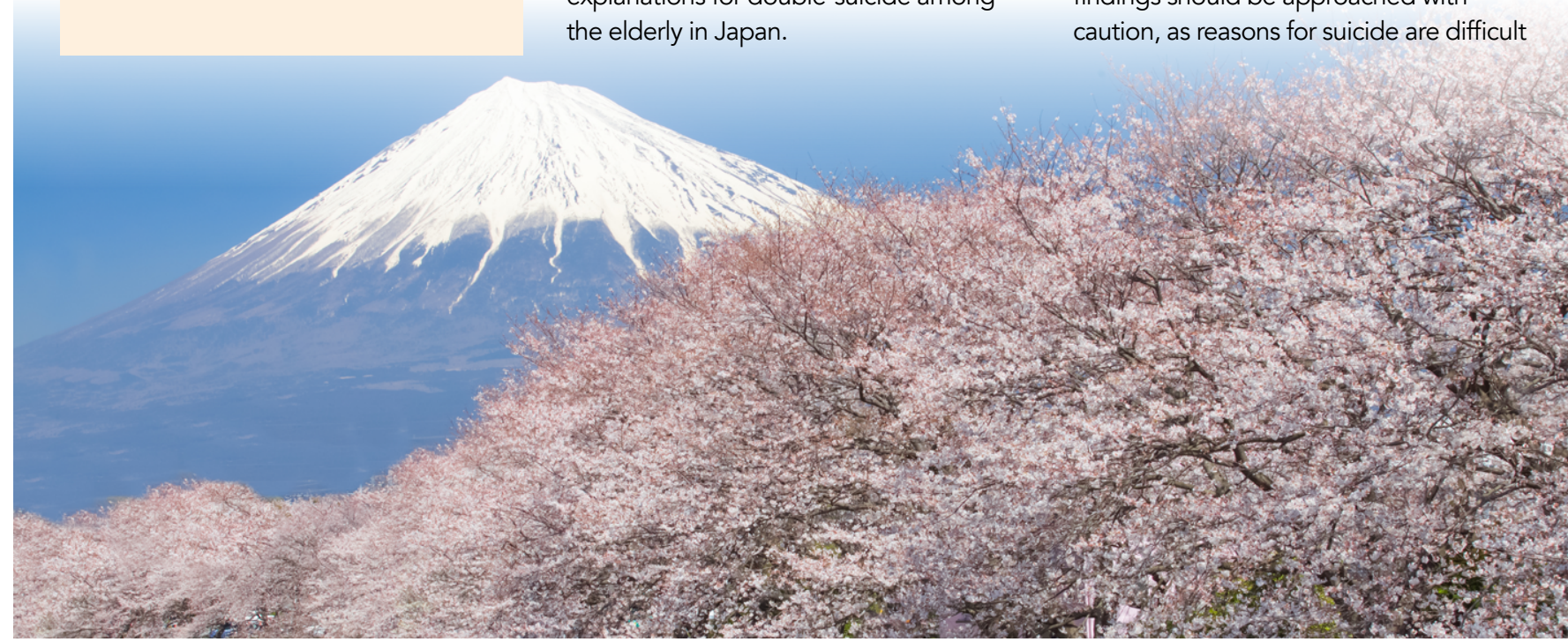

\section{HOMICIDE-SUICIDE} RREOUENCY ESTIMATES Professor Satoh and colleagues extracted data from autopsy reports on unnatu deaths that occurred in Kanagawa as the largest area of Japan in termo Po the largest area of Japan in terms of study homicide-suicide was defined as a suicide case following a killing of a family member, spouse, or lover, and details of demographic and forensic characteristics Overall, there were 76 homicide-suicide cases, involving 169 people, with an average of 5.8 cases of homicide-suicide occurring per year. A third of all cases involved married couples, and two-thirds involved parents and their children with an even gender split between the perpetrators. Motivations were confirmed in $40 \%$ of cases, and included pain from sickness, psychiatric disorder, prosily problems, as well as caregiver and menthe

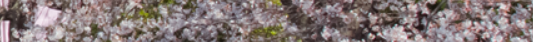
of all eligible cases were examined.

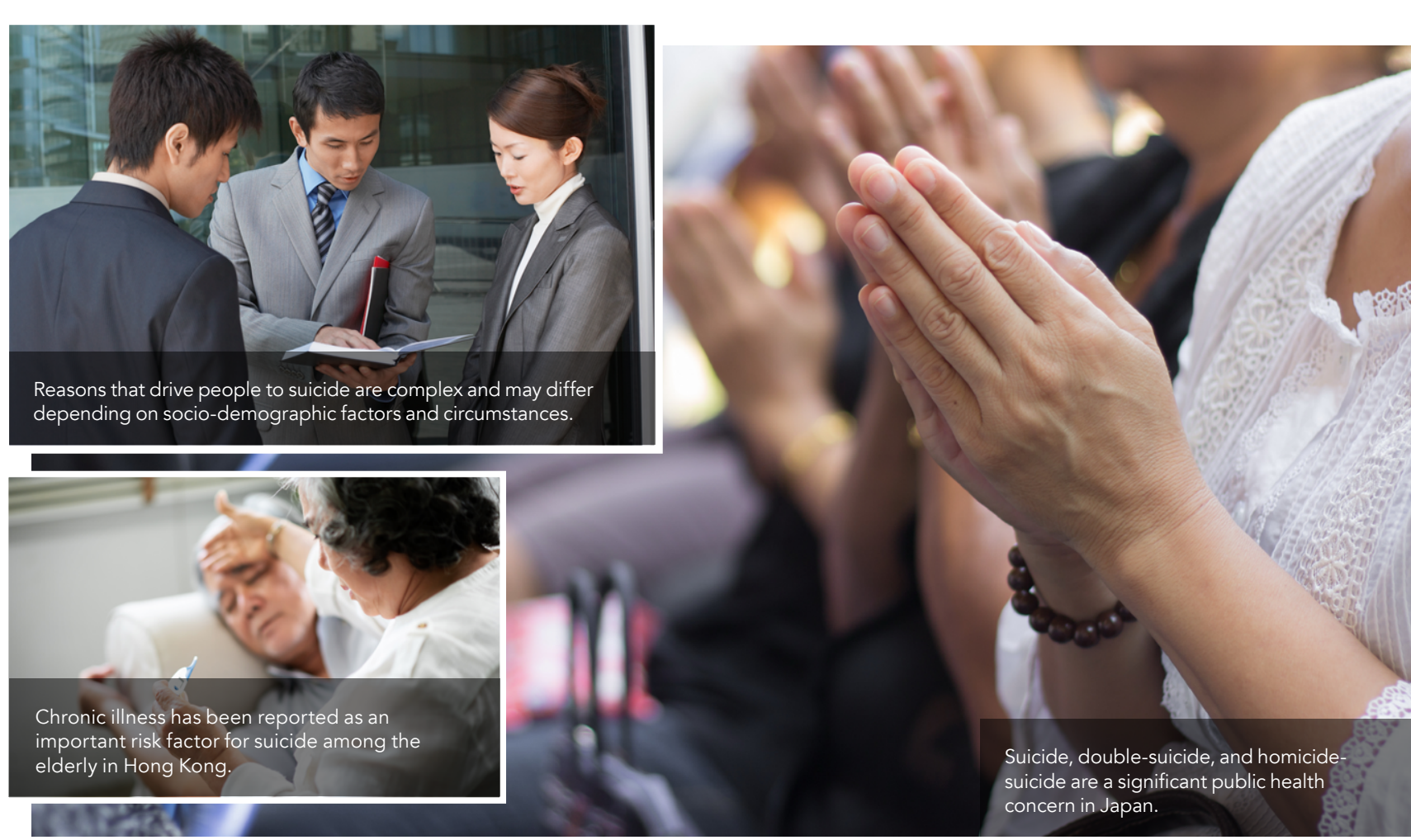

to ascertain and often require in-depth interviews with the surviving bereaved concurrently, previous research has suggested that over half of all homicidedepressed at the time of the event.

Compared to the rest of the world, the suicide rate for Japan in this study was higher than for the United States or the Netherlands, but comparable to Switzerland. In contrast, homicide rate was lower compared to the Netherlan and United States, but comparable to Switzerland. Regarding the homic reported for the United States, with the Netherlands, Switzerland, and Japan reporting a comparable rate The striking differences in the data came when comparing the gender of perpetrators while it was evenly split between genders in Japan, the majority of perpetrators in the United States or Switzerland were male.

\title{
SOCIAL PROBLEM
}

Homicide-suicide within families, such as mothers killing their children ahead of taking their own lives, is a significant socia problem in Japan. At the same time, as the number of the elderly increases, it is a problem that is shifting demographics, crumble

elderly parents or partners. To address this issue, in April 2000, Japan launched a long-term care (LTC) insurance system that the frail to solve the problem of caring care and reducing caregiver burden. The system was expected to decrease the number of murders on insurance recipients committed by their caring kn. However, studies have shown that instead of decreasing, the number of eses in this population in fact

illness), economic and life problems (especially relevant among men), and family problems.

Chronic illness has been reported as an important risk factor for suicide among the elderly in Hong Kong. In addition, depression, the risk of which increases Whinge, has also been reported as a . elderly, suggesting that identifying and

Health, family, and economic problems are some of the forces driving suicide rates among the Japanese elderly.

increased, following the introduction of this system. A systematic review revealed that the most frequent perpetrators of followed by sons or daughters and the parents. The majority of victims have been reported to be bedridden due to stroke or dementia.

\section{REASONS FOR VIOLENC}

Reasons that drive people to suicide are complex and may differ depending on socio-demographic factors and circumstances. In this particular contex health problems (e.g. physical or mental should be high on the public health policy agenda. Policy notwithstanding dealing effectively with depression requires collaboration between physicians, nurses, psychiatrists, and administrative organisations. As social solation, particularly among the frail INing in rural areas, can exacerbate fymptoms of depression and increase risk is

Understanding the other factors that may drive suicide rates among the elderly in Japan requires further research. Som 


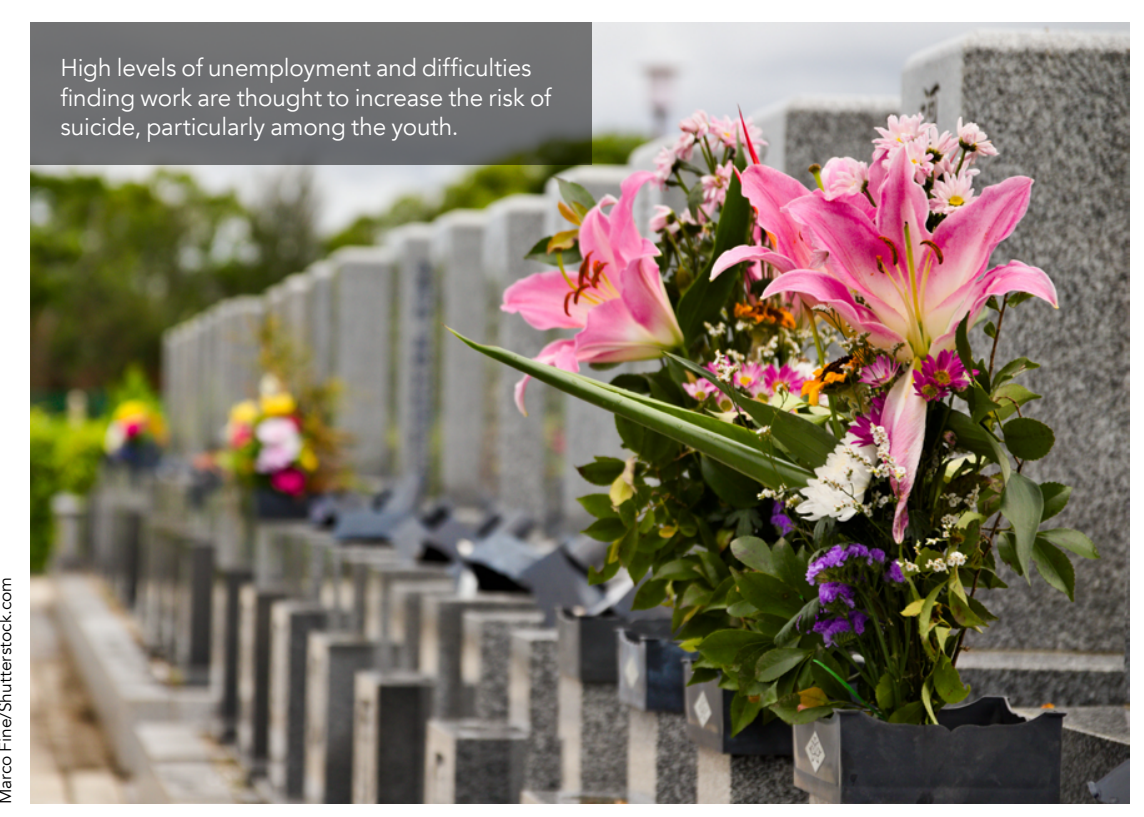

the socio-economic wellbeing of the elderly persons, especially men, might whether this is a independent risk factor or whether this is a factor associated with

\section{ECONOMY AND SUICIDE}

that recession is a risk factorty for suicide. Following the economic downturn of the 1990s, Japan has witnessed a spike in suicide rates, which, unexpectedly, did not begin to decline when the economy began its recovery. High levels of unemployment and difficulties finding

job, compounding the stigma against suicide, particlarly among individus whose social network had been

previously restricted to colleagues. Even among workers who managed to employment, the stress of increased workload and higher expectations combined with lessening of protections had resulted in deaths from overwork.

CULTURAL CONTEXT

OF SUICIDE IN JAPAN

Addressing the burden of care over the elderly is an urgent public health task in Japan, which sits on top of measures

Reasons that drive people to suicide are complex and may differ depending on sociodemographic factors and circumstances.

been suggested to increase the risk of suicide, particularly among the youth. In addition to young people struggling for work, recession has also left many middlle-aged, previously prosperous,
and dedicated men without a steady

suicide rates in the general population. Cultural context has been proposed as set of independent risk factors for suicide in Japan, for example, the collectivist hat prioritises the group over an individual.

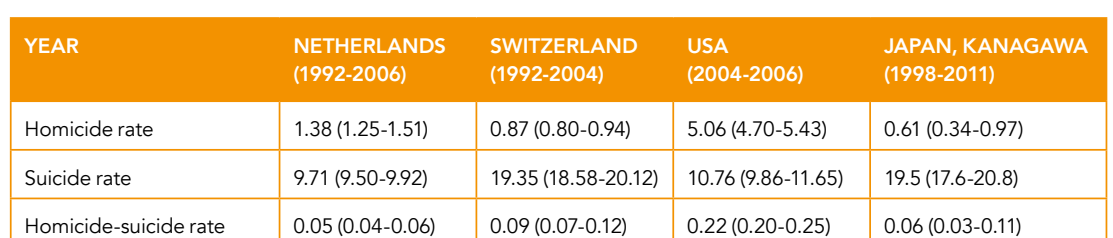

Incidence rates of murder, suicide and homicide-suicide per 100,000 people in the Netherlands,
Switzerland, the USA and Kanagawa, Japan $95 \%$ confidence interval).
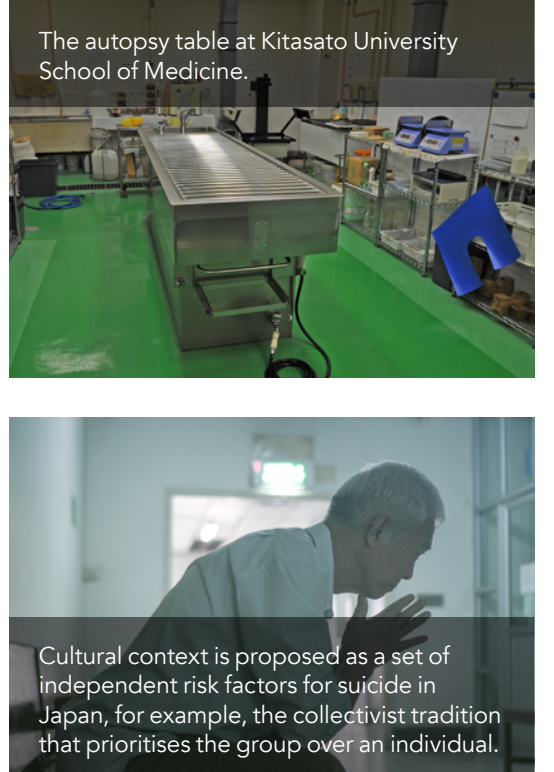

The Japanese are generally very conscious of how they are viewed by their peers, at the expense of personal freed a widespread expectation. Historically, suicide has been viewed as an extreme form of restoring social order, dealing with shame and guilt, and repenting for failures. Suicide has also been romanticised in literature, contributing to permissive attitudes toward it. Recent esearch has suggested that, despite some attitude changes in contemporary Japan Japanese students, compared to their American counterparts, have a higher leve of acceptance for suicide, believing that suicide can be a conscious and acceptab choice. In fact, a separate study has reported that, compared to American respondents, Japanese respondents ide thet opid is acceptable for of the and infirm persons.

\section{SUICIDE COUNTERMEASURES}

\section{Despite a complex landscape that}

may appear conducive toward suicide, taken in Japan. The Support Centre for Suicide Countermeasures was established to implement a comprehensive suicide

prevention strategy, according to the which came into force in April 2016. As a result, regional suicide profiles for each prefecture have been created, leading to suicide rate reduction. Specifically, suicide rate per 100,000 people has decreased by $38.9 \%$ between 2003 and 2018

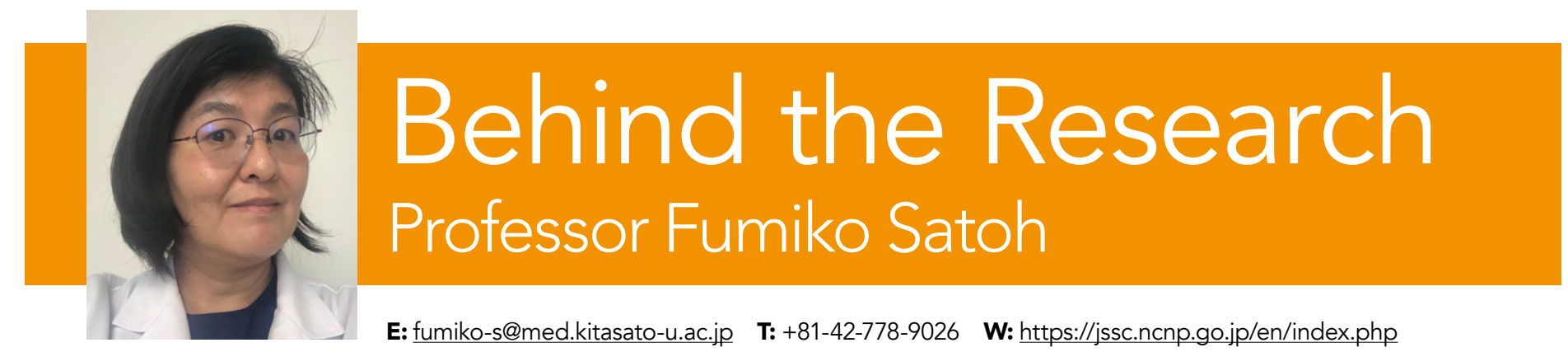

\section{Research Objectives}

As an expert in forensic medicine, Professor Satoh's research transcends the work focuses on sudden natural death, morphological analysis, developing molecular biology analytical systems, sudden infant death syndrome, and the development of drug toxicology analytical assays.

\section{Detail}

Department of Legal Medicine,
Kitasato University School of Medicine

1-15-1, Kitasato, Minami-ku,

Sagamihara, Kanagawa, Japan

Bio

Professor Satoh graduated from Tokai University school of Medicine in 1994. She received her PhD in Medicine in 2000 before working in Legal Medicine for 19 years. She is currently Professor and Chairman at Kitasato University School of Medicine.

\section{Collaborators}

- Motoki Osawa

- Wataru Irie

- Chikako Hashimoto

- Chizu Sasa

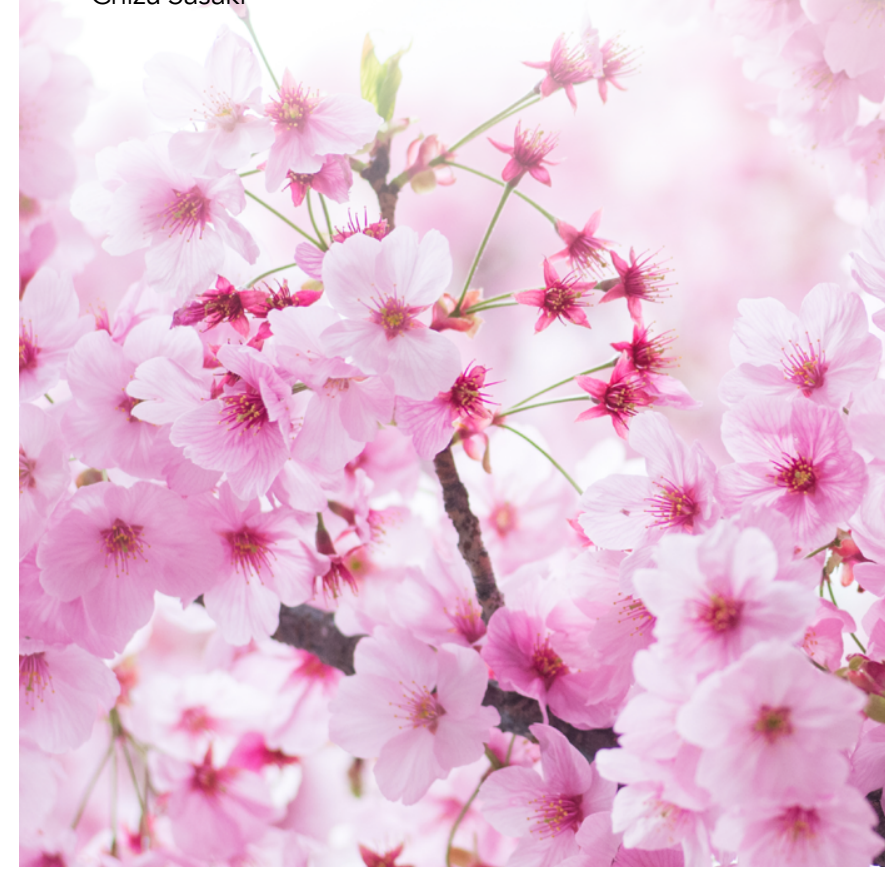

\section{References} Inoue, T., Fukunaga, T., Okazaki, Y,, Ono Y. (2011). Report on
suicide trends in persons aged 60 or over in Japan: the need for effective prevention measures. Med Sci
https://doi.org/10.1258/msl.2010.010068

He, M., Ohrui, T. (2009). Homicides of frail older persons by their caregivers in Japan. J Am Geriatrics Soc, 57(1), 173-174 Russell, R., Metraux, D., Tohen, M. (2016). Cultural influences on suicide in
pcr. 12428

Satoh, F., Osawa, M. (2016). Trend of homicide-

suicide in Kanagawa Prefecture (Japan): Comparison with Western countries. Med Sci
doi:10.1177/0025802416668769

\section{Personal Response}

What do you think is the role of social isolation/ suicide trends in Japan?

III I believe that Japan's nuclear family, diminished hardship all result in increased social isolation. Within this parents and children who are co-dependents on the family or couples, etc. leads to an increased risk from doublesuicide or homicide-suicide. 